Internat. J. Math. \& Math. Sci.

Vol. 23, No. 7 (2000) 497-505

S0161171200001356

(C) Hindawi Publishing Corp.

\title{
EFFECT OF ROTATION AND RELAXATION TIMES ON PLANE WAVES IN GENERALIZED THERMO-VISCO-ELASTICITY
}

\author{
S. K. ROYCHOUDHURI and SANTWANA MUKHOPADHYAY
}

(Received 8 March 1996)

\begin{abstract}
The generalized dynamical theory of thermo-elasticity proposed by Green and Lindsay is applied to study the propagation of harmonically time-dependent thermo-viscoelastic plane waves of assigned frequency in an infinite visco-elastic solid of Kelvin-Voigt type, when the entire medium rotates with a uniform angular velocity. A more general dispersion equation is deduced to determine the effects of rotation, visco-elasticity, and relaxation time on the phase-velocity of the coupled waves. The solutions for the phase velocity and attenuation coefficient are obtained for small thermo-elastic couplings by the perturbation technique. Taking an appropriate material, the numerical values of the phase velocity of the waves are computed and the results are shown graphically to illustrate the problem.
\end{abstract}

Keywords and phrases. Plane waves, rotating visco-elastic medium, generalized thermoelasticity.

2000 Mathematics Subject Classification. Primary 74Dxx.

1. Introduction. The classical theory of thermoelasticity is based on Fourier's law of heat conduction, which predicts an infinite speed of heat propagation. Many new theories have been proposed to eliminate this physical absurdity. Lord and Shulman [4] first modified Fourier's law by introducing into the field equations the term representing the thermal relaxation time. This modified theory is known as the generalized theory of thermoelasticity. Following Lord-Shulman's theory, several authors including Puri [7] and Nayfeh [6] studied the plane thermoelastic wave propagations. Later, Green and Lindsay [3] developed a more general theory of thermoelasticity, in which Fourier's law of heat conduction is unchanged, whereas the classical energy equation and the stress-strain temperature relations are modified by introducing two constitutive constants $\alpha$ and $\alpha^{*}$ having the dimensions of time. Using this theory, Agarwal [1, 2] considered, respectively, thermoelastic and magneto-thermoelastic plane wave propagation in an infinite elastic medium. Later, Mukhopadhyay and Bera [5] applied the generalized dynamical theory of thermoelasticity to determine the distributions of temperature, deformation, stress and strain in an infinite isotropic visco-elastic solid of Kelvin-Voigt type permeated by uniform magnetic field having distributed instantaneous and continuous sources.

Recently, attention has been given to the propagation of thermoelastic plane waves in a rotating medium. Following Lord-Shulman's theory, Puri [8], and Roychoudhuri and Debnath [10] studied plane wave propagation in infinite rotating elastic medium. Roychoudhuri [9] applied Green-Lindsay's theory to study the effect of rotation and 
relaxation time on plane waves in generalized thermoelasticity. Roychoudhuri and Debnath [11] studied magnetoelastic plane wave in a rotating medium too.

In the present paper, the linearized theory of Green and Lindsay having two relaxation times is applied to study the propagation of harmonically time-dependent thermo-visco-elastic plane waves of assigned frequency in an infinite rotating viscoelastic solid of Kelvin-Voigt type. Using the perturbation technique, a dispersion relation for small thermoelastic coupling is obtained to determine the effects of rotation and relaxation times on the phase velocity of the waves in a visco-elastic medium. Numerical values of the wave speeds at various frequencies are computed for an appropriate material and are presented graphically for the purpose of illustration.

2. Formulation of the problem. We consider an infinite isotropic homogeneous visco-elastic solid Kelvin-Voigt type which is rotating uniformly with an angular velocity $\Omega$. The basic field equations in the temperature-rate dependent theory of Green and Lindsay follow (in usual notations).

(i) The stress equations of motion in a rotating medium in the absence of body forces are [12]

$$
\tau_{i j, j}=\rho\left\{\ddot{u}_{i}+[\Omega \times(\Omega \times u)]_{i}+(2 \Omega \times \dot{u})_{i}\right\}, \quad i, j=1,2,3,
$$

where

$$
\tau_{i j}=\left(\lambda_{e}+\lambda_{v} \frac{\partial}{\partial t}\right) \Delta \delta_{i j}+2\left(\mu_{e}+\mu_{v} \frac{\partial}{\partial t}\right) e_{i j}-\gamma(\theta+\alpha \dot{\theta}) \delta_{i j} .
$$

(ii) The heat conduction equation is

$$
K \theta_{, i i}=\rho c_{v}\left(\dot{\theta}+\alpha^{*} \ddot{\theta}\right)+\gamma \theta^{*} \dot{\Delta}, \quad i=1,2,3 .
$$

3. Plane wave solutions and dispersion relation. We consider the waves propagating in the $x$-direction and all the field variables are assumed to be functions of $x$ and time $t$ only. We assume that $u=(u, v, w)$ and $\Omega=(0,0, \Omega)$, where $\Omega$ is a constant. Equation (2.1) with equation (2.2) then reduces to

$$
\begin{gathered}
\rho\left[\ddot{u}-u \Omega^{2}-2 \dot{v} \Omega\right]=\left[\left(\lambda_{e}+2 \mu_{e}\right)+\left(\lambda_{v}+2 \mu_{v}\right) \frac{\partial}{\partial t}\right] \frac{\partial^{2} u}{\partial x^{2}}-\gamma\left(\frac{\partial \theta}{\partial x}+\alpha \frac{\partial^{2} \theta}{\partial x \partial t}\right), \\
\rho\left[\ddot{v}-v \Omega^{2}+2 \dot{u} \Omega\right]=\left(\mu_{e}+\mu_{v} \frac{\partial}{\partial t}\right) \frac{\partial^{2} v}{\partial x^{2}}, \\
\rho \ddot{w}=\left(\mu_{e}+\mu_{v} \frac{\partial}{\partial t}\right) \frac{\partial^{2} w}{\partial x^{2}} .
\end{gathered}
$$

The nondimensional forms of equations (3.1), (3.2), (3.3), and (2.3) are obtained as

$$
\begin{gathered}
\dot{\theta}+\alpha^{*^{\prime}} \ddot{\theta}-\theta^{\prime \prime}+\epsilon_{\theta} \dot{u}^{\prime}=0, \\
{\left[\ddot{u}-u \Omega^{2}-2 \dot{v} \Omega\right]=\left[1+M \frac{\partial}{\partial t}\right] u^{\prime \prime}-\left(\theta^{\prime}+\alpha^{\prime} \dot{\theta}^{\prime}\right),} \\
\beta^{2}\left[\ddot{v}-v \Omega^{2}+2 \dot{u} \Omega\right]=\left(1+N \frac{\partial}{\partial t}\right) v^{\prime \prime}, \\
\beta^{2} \ddot{w}=\left(1+N \frac{\partial}{\partial t}\right) w^{\prime \prime},
\end{gathered}
$$


where we use the following notation and nondimensional variables:

$$
\begin{gathered}
c_{1}^{2}=\frac{\lambda_{e}+2 \mu_{e}}{\rho}, \quad \kappa=\frac{K}{\rho c_{v}}, \quad \epsilon_{\theta}=\frac{\gamma^{2} \theta^{*}}{\rho^{2} c_{v} c_{1}^{2}}, \\
\beta^{2}=\frac{\lambda_{e}+2 \mu_{e}}{\mu_{e}}, \quad M=\frac{\left(\lambda_{v}+2 \mu_{v}\right)}{\left(\lambda_{e}+2 \mu_{e}\right)} \cdot \frac{c_{1}^{2}}{\kappa}, \\
N=\frac{\mu_{v}}{\mu_{e}} \cdot \frac{c_{1}^{2}}{\kappa}, \quad \alpha^{\prime}=\frac{\alpha c_{1}^{2}}{\kappa}, \quad \alpha^{*^{\prime}}=\frac{\alpha^{*} c_{1}^{2}}{\kappa} .
\end{gathered}
$$

$\kappa / c_{1}^{2}, \kappa / c_{1}, \gamma \theta^{*} \kappa / c_{1}^{3} \rho, \theta^{*}, c_{1}^{2} / \kappa$ are taken as the units of time, length, displacement, temperature, and rotation, respectively. Primes denote the differentiation with respect to $x$ and dots denote time differentiation. Equations (3.4), (3.5), and (3.6) form a coupled system and represent coupled visco-thermal-dilatational and shear waves, while equation (3.7) uncouples from the system. This coupling disappears when $\Omega=0$. Thus, the thermal field affects the dilatational and shear motion due to rotation.

4. Dispersion equation for the system. For harmonic solutions of the equations (3.4), (3.5), and (3.6), we choose

$$
(u, v, \theta)=\left(u_{0}, v_{0}, \theta_{0}\right) \cdot \exp \{i(q x+\omega t)\},
$$

where $u_{0}, v_{0}, \theta_{0}$ are amplitude constants, $\omega$ is the prescribed frequency, $q$ is the wave number, in general complex. The phase velocity $c$ and the attenuation coefficients $a$ are then given by

$$
c=\frac{\omega}{\operatorname{Re}(q)}, \quad a=-\operatorname{Im}(q) .
$$

Substituting (4.1) into (3.4), (3.5), and (3.6), we obtain

$$
\begin{gathered}
\left(A_{1} q^{2}-\omega^{2}-\Omega^{2}\right) u_{0}-2 i \omega \Omega v_{0}-\alpha_{1}^{*} \omega q \theta_{0}=0 \\
2 i \omega \Omega \beta^{2} u_{0}+\left(A_{2} q^{2}-\omega^{2} \beta^{2}-\Omega^{2} \beta^{2}\right) v_{0}=0 \\
-\epsilon_{\theta} \omega q u_{0}+\left(q^{2}-\omega^{2} \alpha_{2}^{*}\right) \theta_{0}=0,
\end{gathered}
$$

where

$$
\begin{array}{ll}
\alpha_{1}^{*}=\alpha^{\prime}-\frac{i}{\omega}, & \alpha_{2}^{*}=\alpha^{*^{\prime}}-\frac{i}{\omega}, \\
A_{1}=1+i \omega M, & A_{2}=1+i \omega N .
\end{array}
$$

For the nontrivial solutions for $u_{0}, v_{0}, \theta_{0}$, the dispersion equation of the coupled wave is obtained from (4.3) as

$$
\left(q^{2}-\omega^{2} \alpha_{2}^{*}\right)\left\{\left(A_{1} q^{2}-\Omega_{0}^{2}\right)\left(A_{2} q^{2}-\Omega_{0}^{2} \beta^{2}\right)-4 \Omega^{2} \omega^{2} \beta^{2}\right\}-\epsilon_{\theta} \alpha_{1}^{*} \omega^{2} q^{2}\left(A_{2} q^{2}-\Omega_{0}^{2} \beta^{2}\right)=0,
$$

where $\Omega_{0}^{2}=\Omega^{2}+\omega^{2}$. In case $\Omega=0$, the dispersion equation (4.5) reduces to

$$
\left(A_{2} q^{2}-\omega^{2} \beta^{2}\right)\left\{\left(q^{2}-\omega^{2} \alpha_{2}^{*}\right)\left(A_{1} q^{2}-\omega^{2}\right)-\epsilon_{\theta} \alpha_{1}^{*} \omega^{2} q^{2}\right\}=0 .
$$


On setting $M=0=N$, equations (4.5) and (4.6) agree with [9, equations (3.7) and (3.8)], respectively. Equation (4.5) is therefore a more general dispersion equation in the sense that it incorporates the visco-elastic effect as well as the effects of rotation and relaxation parameters on the propagation of coupled waves. This wave may be called the quasi-visco-elastic-thermal-dilatational-shear wave.

5. Perturbation solution for small $\epsilon_{\theta}$. To obtain the perturbation solution of the dispersion equation for small values of $\epsilon_{\theta}$, we first put $\epsilon_{\theta}=0$ in (4.5) to obtain the following solutions:

$$
q^{2}=\omega^{2} \alpha_{2}^{*}, \quad q_{2,1}^{2}=J_{2,1}^{2},
$$

where

$$
J_{2,1}^{2}=\frac{\left(A_{2}+A_{1} \beta^{2}\right) \Omega_{0}^{2} \pm\left\{\left(A_{2}+A_{1} \beta^{2}\right)^{2} \Omega_{0}^{4}-4 A_{1} A_{2} \beta^{2}\left(\Omega^{2}-\omega^{2}\right)^{2}\right\}^{1 / 2}}{2 A_{1} A_{2}} .
$$

Next, let us write $q^{2}$ in the following forms:

$$
\begin{aligned}
& q_{u}^{2}=q_{1}^{2}+n_{u} \epsilon_{\theta}+0\left(\epsilon_{\theta}^{2}\right), \\
& q_{v}^{2}=q_{2}^{2}+n_{v} \epsilon_{\theta}+0\left(\epsilon_{\theta}^{2}\right), \\
& q_{\theta}^{2}=\omega^{2} \alpha_{2}^{*}+n_{\theta} \epsilon_{\theta}+0\left(\epsilon_{\theta}^{2}\right) .
\end{aligned}
$$

Substituting into equation (4.5), comparing the lowest power of $\epsilon_{\theta}$, and neglecting the terms of $0\left(\epsilon_{\theta}^{2}\right)$, we obtain

$$
\begin{aligned}
& q_{u}^{2}=J_{1}^{2}\left[1+\frac{\omega^{2} \alpha_{1}^{*} \epsilon_{\theta}\left(A_{2} J_{1}^{2}-\beta^{2} \Omega_{0}^{2}\right)}{D_{1}}\right], \\
& q_{v}^{2}=J_{2}^{2}\left[1+\frac{\omega^{2} \alpha_{1}^{*} \epsilon_{\theta}\left(A_{2} J_{2}^{2}-\beta^{2} \Omega_{0}^{2}\right)}{D_{2}}\right], \\
& q_{\theta}^{2}=\omega^{2} \alpha_{2}^{*}\left[1+\frac{\alpha_{1}^{*} \omega^{2} \epsilon_{\theta}\left(A_{2} \alpha_{2}^{*} \omega^{2}-\beta^{2} \Omega_{0}^{2}\right)}{\left[\left(A_{1} \alpha_{2}^{*} \omega_{2}-\Omega_{0}^{2}\right)\left(A_{2} \alpha_{2}^{*} \omega^{2}-\beta^{2} \Omega_{0}^{2}\right)-4 \Omega^{2} \beta^{2} \omega^{2}\right]}\right],
\end{aligned}
$$

where

$$
\begin{aligned}
D_{1,2}= & \left(J_{1,2}^{2}-\omega^{2} \alpha_{2}^{*}\right)\left\{2 A_{1} A_{2} J_{1,2}^{2}-A_{1} \beta^{2} \Omega_{0}^{2}-A_{2} \Omega_{0}^{2}\right\} \\
& +\left(A_{1} J_{1,2}^{2}-\Omega_{0}^{2}\right)\left(A_{2} J_{1,2}^{2}-\beta^{2} \Omega_{0}^{2}\right)-4 \Omega^{2} \beta^{2} \omega^{2} .
\end{aligned}
$$

On putting $M=0=N$, the results (5.2), (5.4), and (5.5) are in agreement with the corresponding results of [9].

6. Determination of wave speeds and attenuation coefficients. From the above solutions, we can observe that the dilatational, shear, and thermal waves propagate in the visco-elastic medium, and these waves are affected by the thermo-visco-elastic coupling coefficient $\epsilon_{\theta}$. Now we find out the wave speed and the attenuation coefficients of the waves for small $\epsilon_{\theta}$. 
I. QUASI-VISCO-THERMAL WAVE. Separating real and imaginary parts, we get from (5.6), for small $\epsilon_{\theta}$,

$$
\begin{aligned}
\operatorname{Re}\left(q_{\theta}\right)= & \omega \sqrt{\frac{L+\alpha^{*^{\prime}}}{2}}+\frac{1}{2} \epsilon_{\theta} \cdot \frac{\omega^{3}}{\sqrt{2}} \cdot \frac{1}{\left(D_{\gamma}^{2}+D_{m}^{2}\right)} \\
& \times\left[\sqrt{L+\alpha^{*^{\prime}}}\left(N_{\gamma} D_{\gamma}+N_{m} D_{m}\right)-\sqrt{L-\alpha^{*^{\prime}}}\left(N_{m} D_{\gamma}-N_{\gamma} D_{m}\right)\right], \\
\operatorname{Im}\left(q_{\theta}\right)= & \omega \sqrt{\frac{L-\alpha^{*^{\prime}}}{2}}+\frac{1}{2} \epsilon_{\theta} \cdot \frac{\omega^{3}}{\sqrt{2}} \cdot \frac{1}{\left(D_{\gamma}^{2}+D_{m}^{2}\right)} \\
& \times\left[\sqrt{L+\alpha^{*^{\prime}}}\left(N_{m} D_{\gamma}-N_{\gamma} D_{m}\right)-\sqrt{L-\alpha^{*^{\prime}}}\left(N_{\gamma} D_{\gamma}+N_{m} D_{m}\right)\right],
\end{aligned}
$$

where

$$
\begin{aligned}
& N_{\gamma}= \alpha^{\prime}\left(\alpha^{*^{\prime}} \omega^{2}+N \omega^{2}-\Omega_{0}^{2} \beta^{2}\right)-\left(1-\alpha^{*^{\prime}} N \omega^{2}\right), \\
& N_{m}=-\left(\alpha^{*^{\prime}} \omega+N \omega-\frac{\Omega_{0}^{2} \beta^{2}}{\omega}\right)-\alpha^{\prime} \omega\left(1-\alpha^{*^{\prime}} N \omega^{2}\right), \\
& D_{\gamma}=\left(\alpha^{*^{\prime}} \omega^{2}+N \omega^{2}-\Omega_{0}^{2}\right)\left(\alpha^{*^{\prime}} \omega^{2}+N \omega^{2}-\Omega_{0}^{2} \beta^{2}\right) \\
&-\omega^{2}\left(1-\alpha^{*^{\prime}} M \omega^{2}\right)\left(1-\alpha^{*^{\prime}} N \omega^{2}\right)-4 \omega^{2} \Omega^{2} \beta^{2}, \\
& D_{m}=-\omega\left[\left(1-\alpha^{*^{\prime}} M \omega^{2}\right)\left(\alpha^{*^{\prime}} \omega^{2}+N \omega^{2}-\Omega_{0}^{2} \beta^{2}\right)\right. \\
&\left.+\left(1-\alpha^{*^{\prime}} N \omega^{2}\right)\left(\alpha^{*^{\prime}} \omega^{2}+M \omega^{2}-\Omega_{0}^{2}\right)\right], \\
& \quad L=\frac{\sqrt{\left\{\left(\alpha^{*^{\prime}} \omega\right)^{2}+1\right\}}}{\omega}, \\
& \sqrt{\alpha_{2}^{*}}=\frac{1}{\sqrt{2}}\left[\sqrt{L+\alpha^{*^{\prime}}}+i \sqrt{L-\alpha^{*^{\prime}}}\right] .
\end{aligned}
$$

Therefore, the thermal wave speed $c_{\theta}=\omega \backslash \operatorname{Re}\left(q_{\theta}\right)$ and the attenuation coefficient $a_{\theta}=-\operatorname{Im}\left(q_{\theta}\right)$, where $\operatorname{Re}\left(q_{\theta}\right)$ and $\operatorname{Im}\left(q_{\theta}\right)$ are obtained above.

II. QUASI-Visco-DilatationAL WAVE. Using (5.2) from (5.4) for small $\epsilon_{\theta}$, the quasi-visco-elastic dilatational wave speed $c_{e}=\omega \backslash \operatorname{Re}\left(q_{u}\right)$ and the attenuation coefficient $a_{e}=-\operatorname{Im}\left(q_{u}\right)$, where

$$
\begin{aligned}
\operatorname{Re}\left(q_{u}\right)=A_{3}+\frac{1}{2} \epsilon_{\theta} \omega \cdot \frac{1}{\left(P^{2}+Q^{2}\right)}\{ & A_{3}[ \\
- & \left.P\left(\alpha^{\prime} \omega K_{3}+K_{4}\right)+Q\left(\alpha^{\prime} \omega K_{4}-K_{3}\right)-Q\left(\alpha^{\prime} \omega K_{3}+K_{3}\right)\right] \\
\left.\operatorname{Im}\left(q_{u}\right)=B_{3}\right)+\frac{1}{2} \epsilon_{\theta} \omega \cdot \frac{1}{\left(P^{2}+Q^{2}\right)}\{ & B_{3}\left[P\left(\alpha^{\prime} \omega K_{3}+K_{4}\right)+Q\left(\alpha^{\prime} \omega K_{4}-K_{3}\right)\right] \\
+ & \left.A_{3}\left[P\left(\alpha^{\prime} \omega K_{4}-K_{3}\right)-Q\left(\alpha^{\prime} \omega K_{3}+K_{4}\right)\right]\right\},
\end{aligned}
$$


where

$$
\begin{aligned}
A_{3} & =\frac{1}{2}\left[R_{3}+\sqrt{R_{3}^{2}+R_{4}^{2}}\right]^{1 / 2}, \quad B_{3}=\frac{1}{2} \frac{R_{4}}{\left|R_{4}\right|}\left[-R_{3}+\sqrt{R_{3}^{2}+R_{4}^{2}}\right]^{1 / 2}, \\
R_{3} & =\frac{\left[\left(1-\omega^{2} M N\right)\left\{\left(\beta^{2}+1\right) \Omega_{0}^{2}-A\right\}+(M+N) \omega \times\left\{\Omega_{0}^{2} \omega\left(N+M \beta^{2}\right)-B\right\}\right]}{\left[\left(1-\omega^{2} M N\right)^{2}+\omega^{2}(M+N)^{2}\right]}, \\
R_{4} & =\frac{\left[\left(1-\omega^{2} M N\right)\left\{\Omega_{0}^{2} \omega\left(N+M \beta^{2}\right)-B\right\}-(M+N) \omega \times\left\{\left(\beta^{2}+1\right) \Omega_{0}^{2}-A\right\}\right]}{\left[\left(1-\omega^{2} M N\right)^{2}+\omega^{2}(M+N)^{2}\right]}, \\
A & =\frac{1}{\sqrt{2}}\left[P_{1}+\sqrt{P_{1}^{2}+P_{2}^{2}}\right]^{1 / 2}, \quad B=\frac{P_{2}}{\left|P_{2}\right|} \cdot \frac{1}{\sqrt{2}}\left[-P_{1}+\sqrt{P_{1}^{2}+P_{2}^{2}}\right]^{1 / 2}, \\
P_{1} & =\left\{\left(\beta^{2}+1\right)^{2}-\omega^{2}\left(N+M \beta^{2}\right)\right\} \Omega_{0}^{4}-4 \beta^{2}\left(1-\omega^{2} M N\right)\left(\Omega^{2}-\omega^{2}\right) \\
P_{2} & =\omega\left[2\left(\beta^{2}+1\right)\left(N+M \beta^{2}\right) \Omega_{0}^{4}-4 \beta^{2}(M+N)\left(\Omega^{2}-\omega^{2}\right)\right] \\
K_{3} & =R_{3}-\beta^{2} \Omega_{0}^{2}-R_{4} \omega M, \quad K_{4}=R_{4}+R_{3} \omega M, \\
E_{3} & =2 R_{3}\left(1-\omega^{2} M N\right)-2 R_{4} \omega(M+N)-\left(\beta^{2}+1\right) \Omega_{0}^{2}, \\
E_{4} & =2 R_{4}\left(1-\omega^{2} M N\right)+2 R_{3} \omega(M+N)-\omega\left(N+M \beta^{2}\right) \Omega_{0}^{2} \\
F_{3} & =R_{3}-R_{4} \omega N-2 \Omega_{0}^{2}, \quad F_{4}=R_{4}+R_{3} \omega N \\
P & =E_{3}\left(R_{3}-\alpha^{*^{\prime}} \omega^{2}\right)-E_{4}\left(R_{4}+\omega\right)+F_{3} K_{3}-F_{4} K_{4}-4 \Omega^{2} \omega^{2} \beta^{2} \\
Q & =E_{3}\left(R_{4}+\omega\right)+E_{4}\left(R_{3}-\alpha^{*^{\prime}} \omega^{2}\right)+F_{4} K_{3}+F_{3} K_{4} .
\end{aligned}
$$

III. QUASI-VISCO-SHEAR WAVE. Using (5.2), we get from (5.5) for small $\epsilon_{\theta}$, the quasivisco-shear wave speed $c_{S}=\omega / \operatorname{Re}\left(q_{v}\right)$ and the attenuation coefficient $a_{S}=-\operatorname{Im}\left(q_{v}\right)$, where

$$
\begin{aligned}
\operatorname{Re}\left(q_{v}\right)= & A_{1}+\frac{1}{2} \epsilon_{\theta} \omega \cdot \frac{1}{\left(P^{\prime 2}+Q^{\prime 2}\right)}\left[A _ { 1 } \left\{P^{\prime}\left(\alpha^{\prime} \omega K_{1}+K_{2}\right)\right.\right. \\
& \left.\left.+Q^{\prime}\left(\alpha^{\prime} \omega K_{2}-K_{1}\right)\right\}-B_{1}\left\{P^{\prime}\left(\alpha^{\prime} \omega K_{2}-K_{1}\right)-Q^{\prime}\left(\alpha^{\prime} \omega K_{1}+K_{2}\right)\right\}\right], \\
\operatorname{Im}\left(q_{v}\right)= & B_{1}+\frac{1}{2} \epsilon_{\theta} \omega \cdot \frac{1}{\left(P^{\prime 2}+Q^{\prime 2}\right)}\left[B _ { 1 } \left\{P^{\prime}\left(\alpha^{\prime} \omega K_{1}+K_{2}\right)\right.\right. \\
& \left.\left.+Q^{\prime}\left(\alpha^{\prime} \omega K_{2}-K_{1}\right)\right\}+A_{1}\left\{P^{\prime}\left(\alpha^{\prime} \omega K_{2}-K_{1}\right)-Q^{\prime}\left(\alpha^{\prime} \omega K_{1}+K_{2}\right)\right\}\right], \\
A_{1}= & \frac{1}{2}\left[R_{1}+\sqrt{R_{1}^{2}+R_{2}^{2}}\right]^{1 / 2}, \quad B_{1}=\frac{R_{2}}{\left|R_{2}\right|} \cdot \frac{1}{2}\left[-R_{1}+\sqrt{R_{1}^{2}+R_{2}^{2}}\right]^{1 / 2}, \\
P^{\prime}= & E_{1}\left(R_{1}-\alpha^{*^{\prime}} \omega^{2}\right)-E_{2}\left(R_{2}+\omega\right)+F_{1} K_{1}-F_{2} K_{2}-4 \Omega^{2} \omega^{2} \beta^{2}, \\
Q^{\prime}= & E_{1}\left(R_{2}+\omega\right)+E_{2}\left(R_{1}-\alpha^{*^{\prime}} \omega^{2}\right)+F_{2} K_{1}+F_{1} K_{2},
\end{aligned}
$$

where $R_{1}$ and $R_{2}$ have the same expressions as $R_{3}$ and $R_{4}$, respectively, with $-A,-B$ in place of $A, B$, respectively, and $E_{1}, E_{2}, K_{1}, K_{2}, F_{1}, F_{2}$ have the same expressions as $E_{3}, E_{4}, K_{3}, K_{4}, F_{3}, F_{4}$, respectively, with $R_{1}$ and $R_{2}$ in place of $R_{3}$ and $R_{4}$, respectively. 
7. Numerical results and discussions. For numerical work, we take copper as the working substance for which

$$
\begin{gathered}
\lambda_{e}=1.387 \times 10^{12} \text { dyne } / \mathrm{cm}^{2}, \quad \mu_{e}=0.448 \times 10^{12} \mathrm{dyne} / \mathrm{cm}^{2}, \\
\rho=8.93 \mathrm{~g} / \mathrm{cm}^{3}, \quad \kappa=1.14 \mathrm{~cm}^{2} / \mathrm{s}, \quad a_{t}=1.67 \times 10^{-8} /{ }^{\circ} \mathrm{c}, \\
\alpha=10^{-11} \mathrm{~s}, \quad \alpha^{*}=2 \times 10^{-11} \mathrm{~s} .
\end{gathered}
$$

We take $\Omega=0.1$.

The numerical computations of the quasi-visco-dilatational wave speed, the quasi-visco-shear wave speed, and the quasi-visco-thermal wave speed for small values of $\epsilon_{\theta}$ are done with the help of a PC and the corresponding graphs are plotted.

The quasi-visco-dilatational wave speed is drawn against the real frequency $\omega$ for three different values of the visco-elastic parameter $M$ in Figure 7.1. The quasi-viscoshear wave speed is plotted against $\omega$ for three different values of $M$ in Figure 7.2 and the quasi-visco-thermal wave speed is drawn against $\omega$ for the same values of $M$ in Figure 7.3.

The variation of different wave speeds with the visco-elastic parameter $M$ can be seen from the graphs. It is observed that although in case of quasi-visco-thermal wave speed (Figure 7.3) the variation is not pronounced appreciably, in cases of the quasi-viscodilatational wave speed (Figure 7.1) and the quasi-visco-thermal-shear wave speed (Figure 7.2) the variations are more pronounced for higher values of $\omega$.

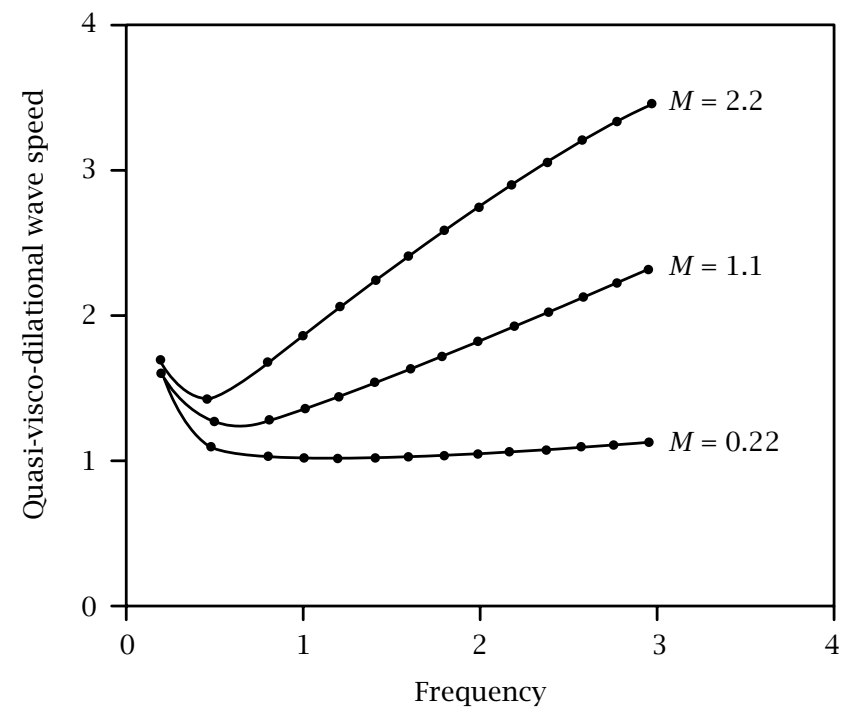

FIGURE 7.1. Plot of quasi-visco-dilatational wave speed against real frequency $\omega$. 


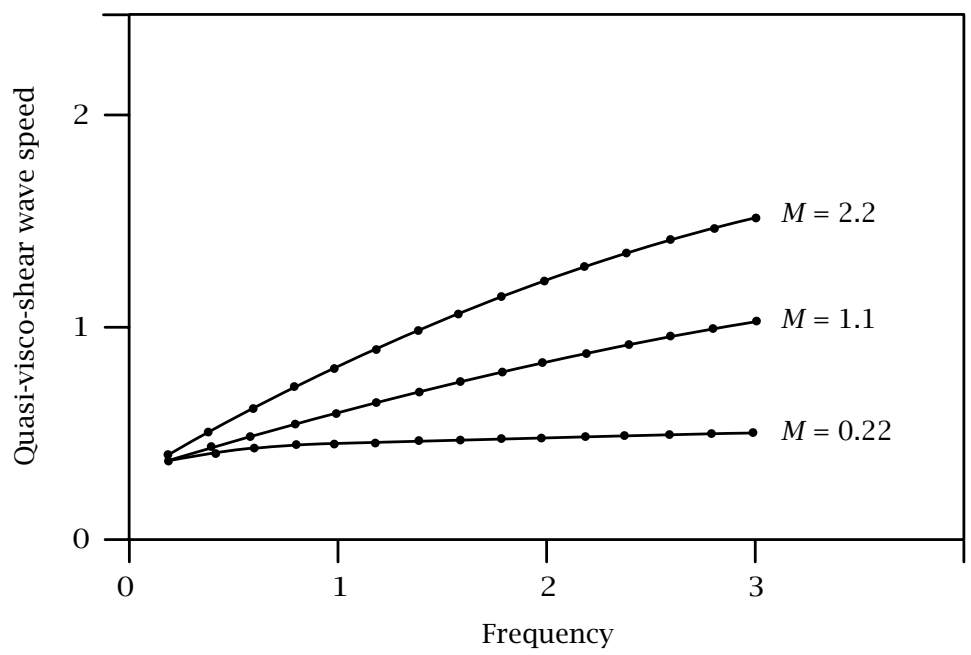

FIGURE 7.2. Plot of quasi-visco-shear wave speed against real frequency $\omega$.

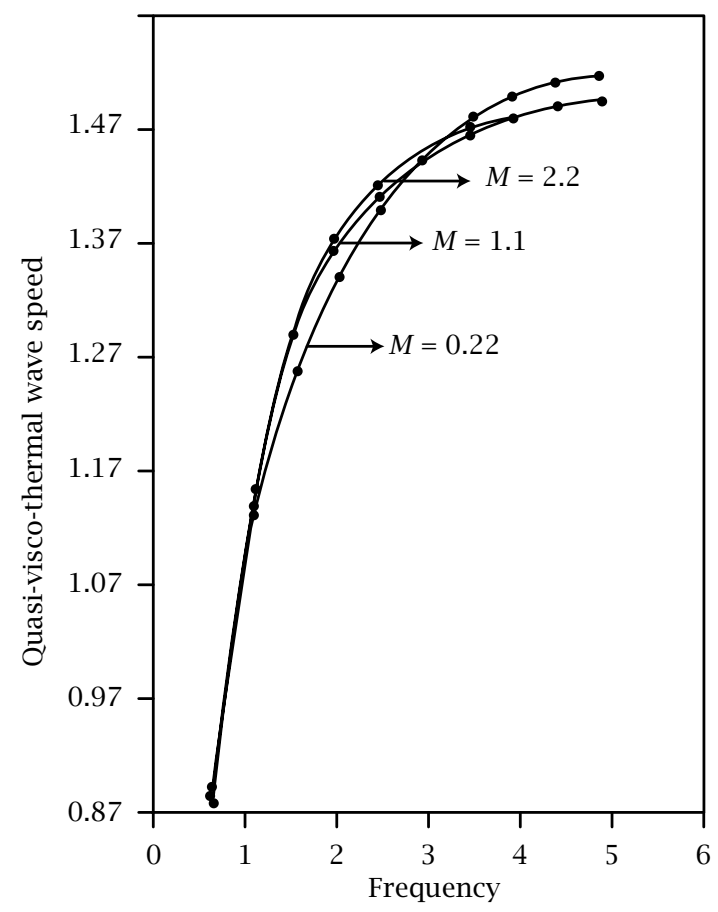

FIGURE 7.3. Plot of quasi-visco-thermal wave speed against real frequency $\omega$.

ACKNOWLEDGEMENT. The authors would like to offer grateful thanks to the Council of Scientific and Industrial Research (CSIR, India) for full financial support. 


\section{REFERENCES}

[1] V. K. Agarwal, On plane waves in generalized thermoelasticity, Acta Mech. 31 (1978/79), no. 3-4, 185-198. MR 80e:73067. Zbl 393.73121.

[2] __ On electromagneto-thermoelastic plane waves, Acta Mech. 34 (1979), no. 3-4, 181191. MR 80m:73067. Zbl 428.73094.

[3] A. E. Green and K. A. Lindsay, Thermoelasticity, J. Elasticity 2 (1972), no. 1, 1-7. Zbl 775.73063.

[4] H. W. Lord and Y. A. Shulman, A generalized dynamical theory of thermoelasticity, J. Mech. Phys. Solids 15 (1967), 299-309. Zbl 156.22702.

[5] B. Mukhopadhyay and R. K. Bera, Effect of distributed instantaneous and continuous heat sources in an infinite conducting magneto-thermo-viscoelastic solid with thermal relaxation, Comput. Math. Appl. 18 (1989), no. 8, 723-728. Zbl 677.73026.

[6] A. Nayfeh and S. Nemat-Nasser, Thermoelastic waves in solids with thermal relaxation, Acta Mech. 12 (1971), 53-69. Zbl 241.73026.

[7] P. Puri, Plane waves in thermoelasticity and magnetothermoelasticity, Internat. J. Engrg. Sci. 10 (1972), 467-476. Zbl 235.73011.

[8]_ Plane thermoelastic waves in rotating media, Bull. Acad. Polon. Sci. Sér. Sci. Tech. 24 (1976), 137-144. Zbl 331.73026.

[9] S. K. Roychoudhuri, Effects of rotation and relaxation times on plane waves in generalized thermoelasticity, J. Elasticity 15 (1985), 59-68. Zbl 558.73016.

[10] S. K. Roychoudhuri and L. Debnath, Magneto-thermo-elastic plane waves in rotating media, Int. J. Engrg. Sci. 21 (1983), no. 2, 155-163. Zbl 501.73011.

[11] _ Magnetoelastic plane waves in infinite rotating media, Trans. ASME Ser. E J. Appl. Mech. 50 (1983), 283-287. Zbl 554.73090.

[12] M. Schoenberg and D. Censor, Elastic waves in rotating media, Quart. Appl. Math. 31 (1973), 115-125. Zbl 281.73018.

ROYCHOUdHURI: DePARTMENT OF MATHEMATICS, UNIVERSITY OF BURDWAN, BURDWAN 713104, INDIA

Mukhopadhyay: Department of Applied Mathematics, Institute of Technology, BANARAS HINDU UNIVERSITY, VARANASI-221 005, INDIA 


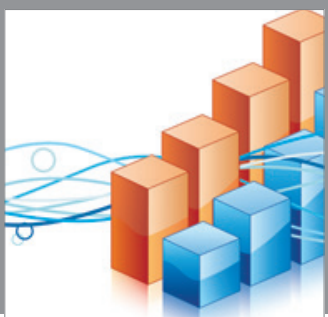

Advances in

Operations Research

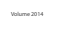

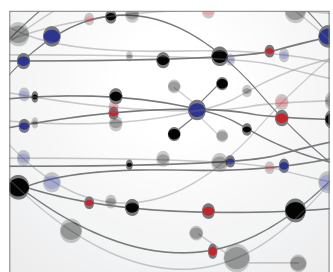

\section{The Scientific} World Journal
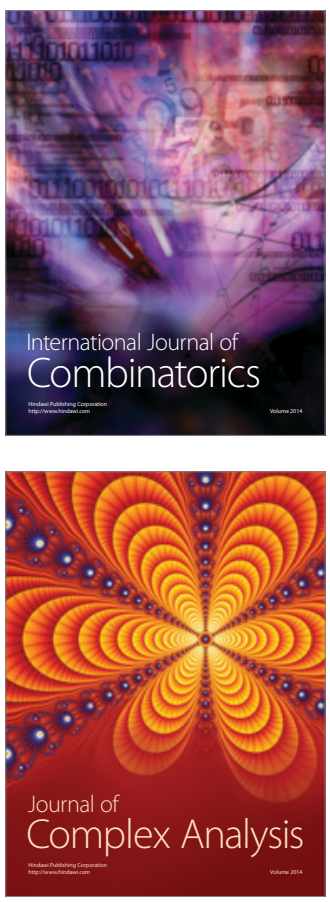

International Journal of

Mathematics and

Mathematical

Sciences
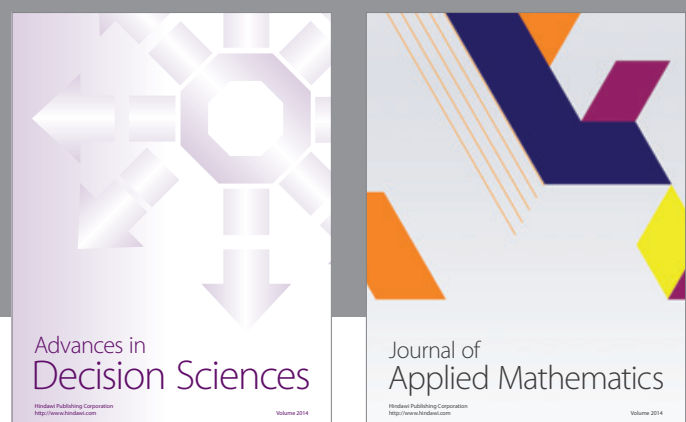

Journal of

Applied Mathematics
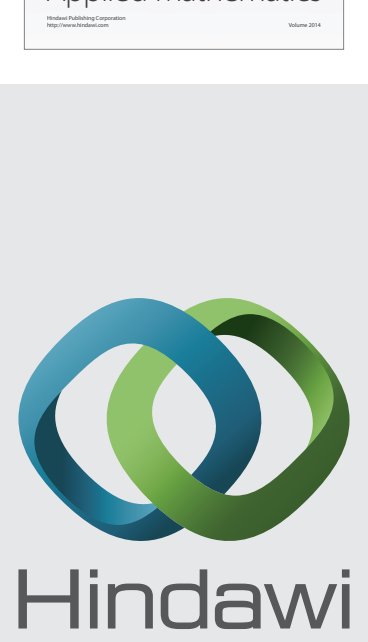

Submit your manuscripts at http://www.hindawi.com
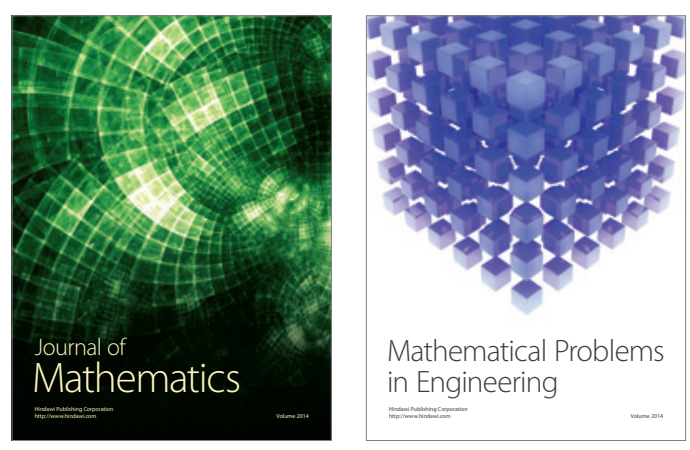

Mathematical Problems in Engineering
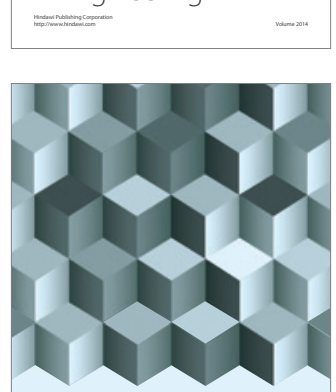

Journal of

Function Spaces
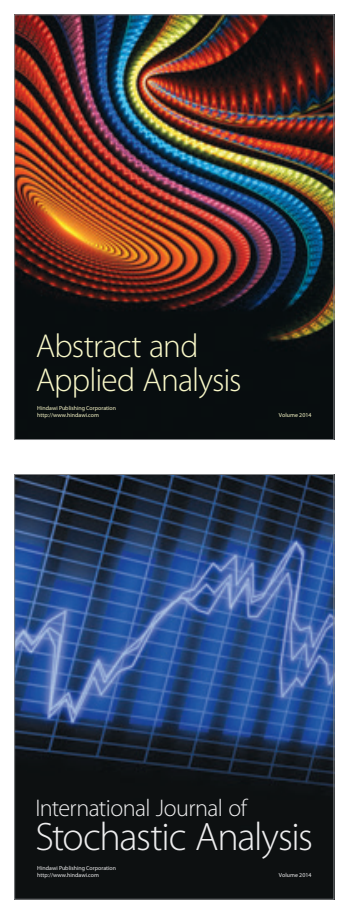

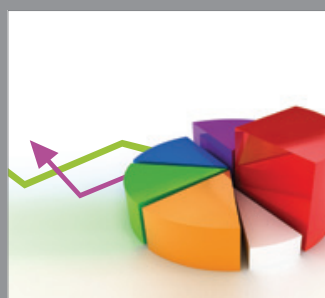

ournal of

Probability and Statistics

Promensencen
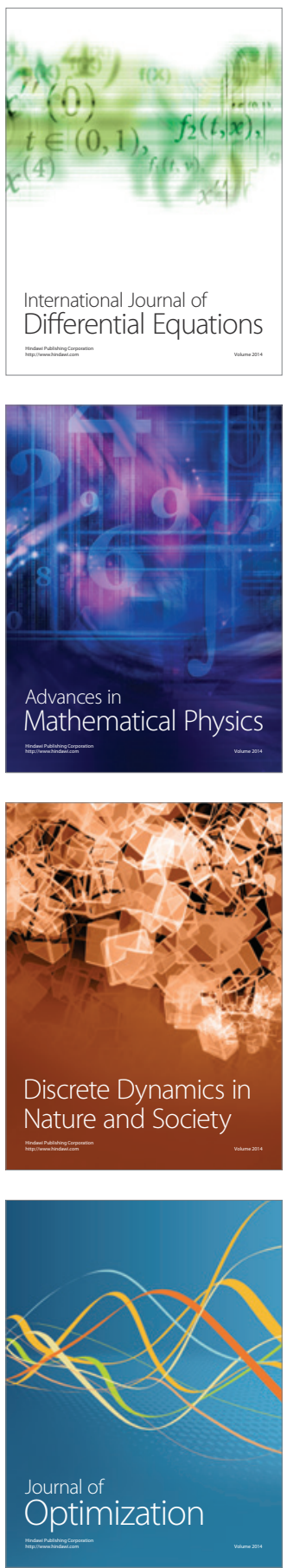